\title{
Indications and Results of Kidney Biopsies in Patients over 65 Years Old
}

\author{
Imen Gorsane, Madiha Mahfoudhi*, Imed Helal, Taieb Ben Abdallah \\ Internal Medicine A Department, Charles Nicolle Hospital, Tunis, Tunisia \\ Email: ${ }^{*}$ madiha_mahfoudhi@yahoo.fr
}

Received 31 March 2015; accepted 7 June 2015; published 10 June 2015

Copyright (C) 2015 by authors and Scientific Research Publishing Inc.

This work is licensed under the Creative Commons Attribution International License (CC BY). http://creativecommons.org/licenses/by/4.0/

(c) (i) Open Access

\begin{abstract}
Normal kidney aging is related to tissue and functional changes that make older patients very vulnerable to environmental modifications. Numerous factors can accelerate the impairment of renal function during aging. It was a single-center retrospective study extending over a period of 30 years from 1984 to 2014 and includes patients older than 65 years, hospitalized for nephropathy requiring renal biopsy and monitored in our service. There were 6 men and 7 women with an average age of $69.38 \pm 4.5$ years. Mean serum creatinine was $267.15 \pm 124 \mathrm{mmol} / \mathrm{l}$. Eleven patients had renal failure. Histological lesions were: Extramembranous glomerulonephritis (EMGN): 1 case, focal segmental glomerular sclerosis (FSGS): 3 cases, membranoproliferative glomerulonephritis (MPGN): 1 case, IgA nephropathy: 1 case, glomerulonephritis with IgG4 fibrillar deposits (GN IgG4): 1 case, diabetic nephropathy: 2 cases, myeloma-associated tubulopathy: 1 case, acute tubular necrosis: 1 case, acute interstitial nephritis: 1 case, vascular nephropathy: 1 case. Kidney disease will increasingly become a geriatric illness. Nephrologists are encountering a growing group of elderly patients with diminished equation for estimated glomerular filtration rate (eGFR) that require evaluation and management.
\end{abstract}

\section{Keywords}

Renal Aging, Chronic Kidney Disease, Elderly, Renal Biopsy

\section{Introduction}

The aging of the current population and future is recognized by all demographic studies. The health professional currently holds the age of 70 to define an old person.

The global population is aging, and the number of people above the age of 70 years is growing faster than any other age group.

*Corresponding author. 
There is an age-related decline in kidney function; however, not all individuals will develop chronic kidney disease with advancing age.

Renal failure in the elderly is currently underestimated. Numerous factors can accelerate the impairment of renal function during aging [1]. We report our experience of care in nephrology in the elderly.

\section{Material and Methods}

It was a single-center retrospective study extending over a period of 30 years from 1984 to 2014 and includes patients older than 65 years, hospitalized for nephropathy requiring renal biopsy and monitored in our service.

We studied their epidemiological clinical and histological profile and the different complications.

The ethics committee had no objections against this study since it reflects our clinical work habits and did not include supplementary measures (other biological or radiological examinations).

We performed a comparative study of comorbidities between the men and women patients.

Baseline characteristics were described as means and standard deviations for continuous variables, and frequencies and proportions for categorical variables.

A study of the correlation was made between the various parameters by the statistical test CHI2. $P$ value $\leq$ 0.05 was regarded as significant.

\section{Results}

There were 6 men and 7 women with an average age of $69.38 \pm 4.5$ years. Four patients were diabetic and 9 hypertensive whose coronary artery disease patient. Three patients had cardiac arrhythmia and 2 had stroke.

Mean serum creatinine was $267.15 \pm 124 \mu \mathrm{mol} / \mathrm{l}$ and the mean plasma clearance of creatinine measured by MDRD was $43.60 \mathrm{ml} / \mathrm{mn}$. Eleven patients had renal failure: Six patients in stage 3, 2 cases in stage 4 and 3 cases in stage 5.

Medium hemoglobin was $11.14 \pm 0.5 \mathrm{~g} / \mathrm{dl}$ and Albumin $29 \pm 1.9 \mathrm{~g} / \mathrm{l}$. the mean serum cholesterol was $3.87 \pm$ $1.06 \mathrm{mmol} / \mathrm{l}$, the mean triglyceride level was $1.34 \pm 0.7 \mathrm{mmol} / \mathrm{l}$. The mean serum calcium was $2.33 \pm 0.18$ $\mathrm{mmol} / \mathrm{l}$ The average phosphorus was $1.39 \pm 0.17 \mathrm{mmol} / \mathrm{l}$, and the PTH $384 \pm 421 \mathrm{pg} / \mathrm{ml}$. The mean proteinuria was $2.5 \pm 0.46$ g per 24 hours (Table 1 ).

A comparative study of comorbidities sex found no significant difference between the 2 groups (Table 2).

Indications of renal biopsy were: nephrotic syndrome in 8 cases, rapidly progressive renal failure in 4 cases and microscopic hematuria in one case.

The different histological lesions were: Extramembranous glomerulonephritis (EMGN): 1 case, focal segmental glomerular sclerosis (FSGS): 3 cases, membranoproliferative glomerulonephritis (MPGN): 1 case, IgA nephropathy: 1 case, glomerulonephritis with IgG4 fibrillar deposits (GN IgG4): 1 case, diabetic nephropathy: 2 cases, myeloma-associated tubulopathy: 1 case, acute tubular necrosis: 1 case, acute interstitial nephritis: 1 case, vascular nephropathy: 1 case (Figure 1).

\section{Table 1. Patient’s clinical and biochemical characteristics.}

\begin{tabular}{cc}
\hline Characteristic & Value \\
\hline Age (years) & $69.38 \pm 4.5$ \\
Sex M/F & $6 / 7$ \\
creatinine ( $\mu \mathrm{mol} / \mathrm{l})$ & $267.15 \pm 124$ \\
Hemoglobin $(\mathrm{g} / \mathrm{dl})$ & $11.14 \pm 0.5$ \\
Albumin (g/l) & $29 \pm 1.9$ \\
cholesterol (mmol/l) & $3.87 \pm 1.06$ \\
triglyceride (mmol/l) & $1.34 \pm 0.7$ \\
calcium (mmol/l) & $2.33 \pm 0.18$ \\
phosphorus (mmol/l) & $1.39 \pm 0.17$ \\
PTH (pg/ml) & $384 \pm 421$ \\
Proteinuria (g per 24 hours) & $2.5 \pm 0.46$ \\
\hline
\end{tabular}

M: male, F: female, PTH: parathormone. 


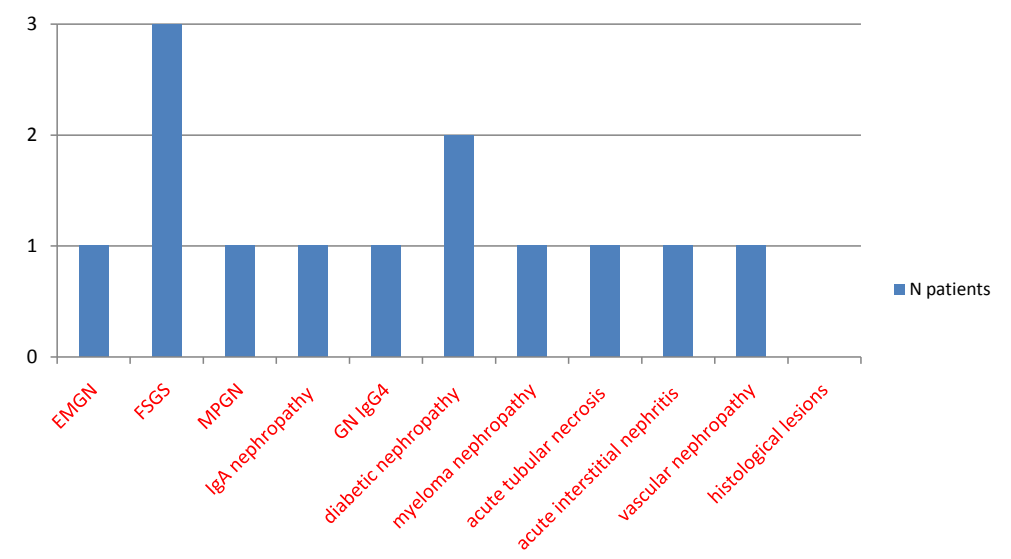

Figure 1. Graphical presentation of histological lesions of our patients.

Table 2. Comparison of comorbidities between the male and female patients.

\begin{tabular}{cccc}
\hline & M (N = 6) & W (N = 7) & P \\
\hline Diabetes (\%) & $1(16.66)$ & $3(42.85)$ & 0.12 \\
Hypertension (\%) & $5(83.33)$ & $4(57.14)$ & 0.5 \\
coronaropathy (\%) & $1(16.66)$ & $2(28.57)$ & 0.3 \\
cardiac arrhythmia (\%) & $1(16.66)$ & $0(0)$ & 0.1 \\
stroke (\%) & $1(16.66)$ & $15(14.28)$ & 0.4 \\
poor mobility (\%) & $1(16.66)$ & $4(57.14)$ & 0.5 \\
\hline
\end{tabular}

All patients had a specific treatment and the outcome was favorable for 10 patients.

The 3 patients who were immediately in stage 5 have not recovered, 2 were in hemodialysis and 1 in peritoneal dialysis.

Two patients died from severe bronchopneumopathy.

\section{Discussion}

From 40 years, the aging kidney results in a reduced renal mass, kidney size $(-0.8 \% /$ year $)$ and the number and the surface of the glomeruli are also observed a reduction in the length of the proximal tubule and dilatation of distal convoluted tubules. Aging functional, that is to say the change in the hemodynamic renal precedes the decreased renal mass.

Reduced mortality and morbidity, prolongation of the patient's life, and better clinical outcomes of renal replacement therapy (RRT) allowed having older patients on hospital nephrology unit for nephropathy.

The prevalence of moderate to severe Chronic Kidney Disease (CKD) increases with age [2].

Renal failure in the elderly is considered as a public health problem. Systematic application of mathematical formulae to estimate the glomerular filtration rate (GFR) of the general population, according to The Kidney Disease Outcomes Quality Initiative (KDOQI) classification of CKD, has permitted to calculate its high prevalence [3]. The MDRD Study equation is the most thoroughly validated equation in the elderly and has shown good performance for patients with all common causes of kidney disease.

Moderate reductions in eGFR can occur as the result of normal aging and should not be equated with CKD in the absence of other abnormalities or clearly defined associated risks [4] [5]. As a result, elderly patients may be mislabeled as having moderate CKD even when their eGFR corrected for age is normal [6].

Kidney biopsies are increasingly being performed in the elderly [7]. Proteinuria is the main sign of kidney injury [8]. Different spectrum of pathologies is found in old population and requires a careful assessment of risks and benefits of any potential therapeutic intervention. 
Common findings are age-related kidney fibrosis related to increased collagen accumulation and advanced vascular changes, similar to chronically damaged kidneys [7] [9].

Primitive nephropathies frequently are represented by the EMGN. Regarding secondary nephropathies, vasculitis pauci immune remain largely the most frequent etiologies [10]. The kidney biopsy in the elderly remains an essential tool to establish a precise histological diagnosis and guide decision for therapeutic management.

Specific treatment of certain nephropathy based on corticosteroids and immunosuppressive with an increased risk of secondary effects in the elderly population because of their immune status related to age with increased risk of infections. Moreover, pharmacodynamics/pharmacokinetics of drugs becomes different with age and the nephrologist has to be aware of the effect of the multiple other drugs these patients are taking. This is too common in this population and is more likely to cause drug-drug interactions and serious adverse effects [11]. In our series two patients died from severe bronchopneumopathy probably due to the state of immunosuppression induced by immunosuppressive treatment.

Older patients with kidney disease often have considerable comorbidity, not only the vascular disease associated with their renal disease, but also the comorbidity found in many older people, including impaired vision, deafness, poor mobility, arthritis and cognitive dysfunction. They are often socially isolated, live in poor accommodations and may have financial problems [12] [13].

Elderly patients can use avoidance or denial based techniques. This was observed where several primitive strategies were being used, most notably denial [14]. Denial is an effective strategy in coping with illness-related stress, but may result in lower compliance with treatment [15].

The elderly patients are depressed and often avoid personal involvement, which may result in difficulties in treatment and physical rehabilitation. The role of a psychologist should be to guide elderly patients toward treatment and lifestyle that may result in substantial benefits from both the patients' and clinics' point of view [16].

However, less is known about how nephrologists prepare patients for understanding their individual prognoses and living with their kidney disease; especially when the patients are aged [17].

Our study, which recruited cases over a long period (30 years), but whose membership is low, did not draw significant conclusions; further studies with larger numbers are needed to have valid results.

\section{Conclusions}

Since the incidence and prevalence of kidney disease increase with advancing age, nephrologists are increasingly confronted with a population of patients who are elderly and have a large number of comorbid conditions requiring ongoing care.

A comparative study of comorbidities sex found no significant difference between the two groups probably because of the small number of our series.

\section{Conflict of Interest}

There are no conflicts of interest.

\section{References}

[1] Bolignano, D., Mattace-Raso, F., Sijbrands, E.J. and Zoccali, C. (2014) The Aging Kidney Revisited: A Systematic Review. Ageing Research Reviews, 14, 65-80. http://dx.doi.org/10.1016/j.arr.2014.02.003

[2] James, M.T., Hemmelgarn, B.R. and Tonelli, M. (2010) Early Recognition and Prevention of Chronic Kidney Disease. The Lancet, 375, 1296-1309. http://dx.doi.org/10.1016/S0140-6736(09)62004-3

[3] Heras, M., Fernández-Reyes, M.J., Guerrero, M.T., Sánchez, R., Muñoz, A., Macías, M.C., et al. (2009) Elderly Patients with Chronic Kidney Disease: What Happens after 24 Months of Follow-Up? Nefrologia, 29, 343-349.

[4] Campbell, K.H. and O’Hare, A.M. (2008) Kidney Disease in the Elderly: Update on Recent Literature. Current Opinion in Nephrology \& Hypertension, 17, 298-303. http://dx.doi.org/10.1097/MNH.0b013e3282f5dd90

[5] Roderick, P.J., Atkins, R.J., Smeeth, L., Mylne, A., Nitsch, D.D., Hubbard, R.B., et al. (2009) CKD and Mortality Risk in Older People: A Community-Based Population Study in the United Kingdom. American Journal of Kidney Diseases, 53, 950-960. http://dx.doi.org/10.1053/j.ajkd.2008.12.036

[6] Anderson, S., Halter, J.B., Hazzard, W.R., Himmelfarb, J., Horne, F.M., Kaysen, G.A., et al. (2009) Prediction, Progression and Outcomes of Chronic Kidney Disease. Journal of the American Society of Nephrology, 20, 1199-1209. http://dx.doi.org/10.1681/ASN.2008080860 
[7] Moutzouris, D.A., Herlitz, L., Appel, G.B., Markowitz, G.S., Freudenthal, B., Radhakrishnan, J., et al. (2008) Renal Biopsy in the Very Elderly. Clinical Journal of the American Society of Nephrology, 4, 1073-1082. http://dx.doi.org/10.2215/CJN.00990209

[8] García de Vinuesa, S. (2008) Progression Factors for Chronic Kidney Disease. Secondary Prevention. Nefrologia, S3, 17-21.

[9] Rodriguez-Puyol, D. (1998) Nephrology Forum. The Aging Kidney. Kidney International, 54, 2247-2265. http://dx.doi.org/10.1038/4499994

[10] Celik, B., Yaz, M., Bulut, T., Sahin, S., Alp, A. and Meteoglu, I. (2013) Is Focal Segmental Glomerulosclerosis Common among the Elderly? Geriatric Biopsy Results. Saudi Medical Journal, 34, 760-763.

[11] Rosner, M., Abdel-Rahman, E. and Williams, M.E. (2010) Geriatric Nephrology: Responding to a Growing Challenge. Clinical Journal of the American Society of Nephrology, 5, 936-942. http://dx.doi.org/10.2215/CJN.08731209

[12] Brown, E.A., Johansson, L., Farrington, K., Gallagher, H., Sensky, T., Gordon, F., et al. (2010) Broadening Options for Long-Term Dialysis in the Elderly (BOLDE): Differences in Quality of Life on Peritoneal Dialysis Compared to Haemodialysis for Older. Nephrology Dialysis Transplantation, 25, 3755-3763. http://dx.doi.org/10.1093/ndt/gfq212

[13] Foote, C., Ninomiya, T., Gallagher, M., Perkovic, V., Cass, A., McDonald, S.P., et al. (2012) Survival of Elderly Dialysis Patients Is Predicted by Both Patient and Practice Characteristics. Nephrology Dialysis Transplantation, 27, 3581-3587. http://dx.doi.org/10.1093/ndt/gfs096

[14] Telford, K., Kralik, D. and Koch, T. (2006) Acceptance and Denial: Implications for People Adapting to Chronic Illness: Literature Review. Journal of Advanced Nursing, 55, 457-464. http://dx.doi.org/10.1111/j.1365-2648.2006.03942.x

[15] Jadoulle, V., Hoyois, P. and Jadoul, M. (2005) Anxiety and Depression in Chronic Hemodialysis: Some Somatopsychic Determinants. Clinical Nephrology, 63, 113-118. http://dx.doi.org/10.5414/CNP63113

[16] Laudański, K., Nowak, Z. and Niemczyk, S. (2013) Age-Related Differences in the Quality of Life in End-Stage Renal Disease in Patients Enrolled in Hemodialysis or Continuous Peritoneal Dialysis. Medical Science Monitor, 19, 378-385. http://dx.doi.org/10.12659/MSM.883916

[17] Schell, J.O., Patel, U.D., Steinhauser, K.E., Ammarell, N. and Tulsky, J.A. (2012) Discussions of the Kidney Disease Trajectory by Elderly Patients and Nephrologists: A Qualitative Study. American Journal of Kidney Diseases, 59, 495503. http://dx.doi.org/10.1053/j.ajkd.2011.11.023

\section{Abbreviations}

Chronic Kidney Disease (CKD)

Equation for estimated glomerular filtration rate (eGFR)

Extramembranous glomerulonephritis (EMGN)

Focal segmental glomerular sclerosis (FSGS)

Glomerular filtration rate (GFR)

Glomerulonephritis with IgG4 fibrillar deposits (GN IgG4)

Kidney Disease Outcomes Quality Initiative (KDOQI)

Membranoproliferative glomerulonephritis (MPGN)

Modification of the Diet in Renal Disease (MDRD)

Parathyroid hormone (PTH)

Renal replacement therapy (RRT) 